\title{
Transitional-aged youth perceptions of influential factors for substance-use change and treatment seeking
}

This article was published in the following Dove Press journal:

Patient Preference and Adherence

Number of times this article has been viewed

\author{
Anna Bowers ${ }^{1,2}$ \\ Kristin Cleverley ${ }^{1,2}$ \\ Claudia Di Clemente' \\ Joanna Henderson' \\ 'Centre for Addiction and Mental \\ Health, ${ }^{2}$ University of Toronto, \\ Toronto, ON, Canada
}

Correspondence: Joanna Henderson Centre for Addiction and Mental Health, 80 Workman Way, Toronto, ON M6J IH4, Canada

Tel +I 416535850 I ext 34959

Email joanna.henderson@camh.ca
Abstract: There is an evident disparity between the number of youth who report experiencing problematic substance use and the number who seek treatment. To address this disparity, it is important to understand the reasons youth do and do not seek substance use treatment. Using qualitative data obtained from semistructured interviews with 31 youth aged 17-25 years presenting for treatment at a mental health hospital, the current study identifies themes in the factors that youth identify as having influenced them to seek or delay treatment. In alignment with self-determination theory, youth identified internal factors, such as wanting to better their academic, social, or financial situation, and external factors, such as familial pressure, as motivating them to seek treatment. Factors beyond those encompassed by self-determination theory were also revealed as having influenced youth decisions to seek treatment for substance abuse. These predominantly included structural factors, including satisfaction with previous treatment, accessibility of services, and availability of clinicians. These findings provide important insight for first-contact professionals and service providers looking to enhance youth motivation to seek and engage in treatment. Limitations and opportunities for future research are discussed.

Keywords: substance use, mental health, youth, treatment seeking, motivation

\section{Introduction}

\section{Youth drug and alcohol use}

The treatment-seeking process of youth with substance-use problems has garnered the attention of service providers, researchers, and policy-makers to understand better how to facilitate early access to treatment. Rates of alcohol and drug use increase significantly during high school and postsecondary. ${ }^{1}$ During emerging adulthood, a developmental period between the ages of 18 and 25 years marked by increased autonomy and exploration of interests and relationships and decreased dependence on individual and organization supports, ${ }^{2}$ youth are more likely to have a substance-use disorder than during any other stage of development. ${ }^{3}$ In Canada, approximately $6 \%$ of males and 3\% of females aged 15-24 years have symptoms consistent with substance-use disorder $^{4}$ and $3 \%$ of youth meet diagnostic criteria for a concurrent disorder. ${ }^{5}$

Drug and alcohol use across the life span poses a serious public health problem and often results in negative personal, familial, and societal consequences. ${ }^{6}$ The cost of illicit drug use is nearly US\$200 billion dollars each year to cover health care, lost productivity, incarceration, and drug enforcement. ${ }^{7}$ Onset of substance use during adolescence or emerging adulthood increases the likelihood of severe substance abuse in adulthood and can have negative consequences on interpersonal relationships, 
employment, and physical and mental health. ${ }^{8}$ On a positive note, participation in treatment within the first 10 years of substance-use onset enhances the likelihood of successful maintenance of abstinence. ${ }^{9}$ Additionally, treatment utilization has the potential to reduce the negative outcomes associated with problematic substance abuse, including social maladjustment and psychiatric and family problems. ${ }^{10}$

Given the benefits of early engagement in treatment, the discrepancy between the number of youth experiencing problematic substance use and the number engaging in treatment is concerning. ${ }^{11}$ An important barrier to effective treatment is poor treatment capacity to engage youth. ${ }^{12}$ Accordingly, it is important to understand better the factors that influence or delay youth to seek substance-use treatment. This has important implications for effectively responding to the needs and concerns of youth when they are first thinking about getting help for problematic substance use.

\section{Treatment seeking}

Despite the high prevalence and adverse consequences of alcohol and substance abuse, few youth seek treatment. ${ }^{11}$ The Ontario Student Drug Use and Health Survey found that although $16 \%$ of grade $7-12$ students could benefit from treatment, only $0.6 \%$ had pursued services in the past year. ${ }^{13}$ There are a variety of factors that have been found to explain this gap in youth treatment needs, and help to explain what influences or delays youth to seek treatment. Prior research has uncovered influential personal factors, including youth perceptions of having a problem or needing professional help, ${ }^{14}$ positivity of feelings toward family, ${ }^{15}$ and feeling competent and confident to express ideas and feelings. ${ }^{16}$ Involvement in risky behavior, a recent arrest, and psychological problems, such as a mood disorder, can also prompt or deter youth from seeking substance-use treatment. ${ }^{17-19}$ With regard to social factors, previous studies have revealed that support from peers and family members can influence treatment seeking. ${ }^{20}$ In addition, Goodman et $\mathrm{al}^{21}$ examined the role of social pressure in substance-use treatment and found that peer pressure prompted guilt about substance use and commitment to a treatment program, while family pressure was related to giving into coercion and demands. Interacting with peers who use substances and having family members who use substances has also been found to influence perceptions of the need for treatment. ${ }^{22}$ Structural factors that have been found to delay treatment onset include difficulties with scheduling appointments and trust in and acceptance of the treatment and service provider. ${ }^{23}$ Rapp et $\mathrm{al}^{24}$ also found that treatment availability and ease of admission were important treatment-seeking predictors. Notably, it is also important to recognize that a significant number of youth reduce their substance use and recover without formal treatment. ${ }^{25}$ These findings reveal the importance of considering personal, social, and structural factors that influence treatment seeking. The perceptions of youth with regard to factors within these domains have yet to be examined, and are important for better understanding and addressing the low proportion of youth with problematic substance use who engage in treatment.

The exploration of motivation and readiness to change among youth with substance-use challenges has gained more attention from researchers and clinicians in the last decade. ${ }^{26}$ Motivation is an important factor when considering treatment seeking, engagement, and outcomes. ${ }^{27}$ Specifically, internal motivation involves doing something for its own sake without concern for the results of the action. ${ }^{28}$ In the case of treatment for substance-use problems, it involves both a desire to change and a readiness to change in either the presence or absence of treatment. ${ }^{20}$ Although treatments are often designed for individuals who are internally motivated to take action and change, many youth seek or enter treatment as a result of external pressure from family members, friends, employers, or the criminal justice system..$^{20,21}$ External motivation may prompt the youth to seek treatment and influence the development of internal motivation once they are in treatment, though long-term recovery is strongly dependent on intrinsic motivational factors. ${ }^{29}$

Self-determination theory (SDT) serves as a helpful framework to examine the factors that influence youth to seek treatment or delay seeking treatment. This theory aims to understand human motivation and behavior through the concepts of innate, universal, and psychological needs. ${ }^{28}$ For example, youth may feel motivated to seek treatment to feel more connected or in control, whereas other youth may be motivated to delay seeking treatment to retain a sense of autonomy. SDT proposes that motivational states range along a continuum from acting in response to external pressure to being internally motivated to take action out of self-interest. ${ }^{30}$ This is particularly relevant to youth, as many enter treatment because it has been mandated or recommended, as opposed to self-referral. SDT proposes that there is a varying degree of overlap between external pressures and autonomous drive. ${ }^{31}$ The combination of one's own feelings and desires to change, as well as one's perceptions of external pressures, is thought to determine an individual's likelihood of engaging in a particular action. ${ }^{32}$ In the context of this study, SDT can help in understanding of youth perceptions 
of internal and external influences that prompt or delay the treatment-seeking process. In addition, this study aims to highlight factors beyond individual and external motivations and behaviors as proposed by SDT that influence youth to change their substance use.

Overall, the literature highlights that although negative outcomes of substance use and positive treatment outcomes have been revealed among youth, only a small proportion seek treatment. There is a need to understand influential factors for seeking or delaying treatment from a youth's perspective better, to facilitate entry into treatment and enhance the likelihood of improved functioning and quality of life.

\section{Present study}

Understanding how youth describe their experience seeking substance-use treatment has important implications for clinical practice, particularly with regard to ensuring that barriers to and facilitators for treatment seeking are considered in treatment engagement and planning. This study expands on the research conducted by Goodman et $\mathrm{al}^{2}$ exploring how the transition to adulthood impacts and is impacted by treatment seeking, as well as research by Henderson et $\mathrm{al}^{33}$ examining youth perceptions of parental influences on motivation to seek treatment. The current study investigated the following research questions. What factors do youth identify as having influenced them to seek or delay treatment? How can these factors be understood in relation to one another? How do these factors align with or differ from SDT? A qualitative thematic analysis design was used to guide the initial coding and identification of themes. The analysis extended the framework of SDT to allow for confirmation and new evidence to emerge.

\section{Materials and methods}

The qualitative thematic analysis approach was used in the collection, analysis, and description of findings from the data for this study. This method allows for a flexible approach to providing a complex and detailed account of the data by identifying, analyzing, and reporting themes and describing a data set. ${ }^{34}$ By examining participants' realities, meanings, and experiences, qualitative thematic analysis facilitates an enhanced understanding of a topic that in turn has important implications for researchers, service providers, and policymakers. ${ }^{35}$ The study protocol was submitted and approved by the Centre for Addiction and Mental Health Research Ethics Board.

Participants were recruited using flyers put up in the youth addiction-services area within a mental health hospital and handed out by clinicians in the hospital directly to clients. Interested youth were connected to research staff to set up an appointment for the interview. Eligibility criteria were English-speaking, between the ages of 16 and 25 years, currently seeking services at the mental health hospital, and no need for immediate psychiatric services. The final sample consisted of 31 youth (16 females, 14 males, one unreported; ages 17-25 years, mean age 20.8 years) who provided consent to participate. Over $90 \%$ of youth were Canadian-born and English-speaking. The majority of youth lived at home with their parents $(71 \%)$, and the remaining participants lived on their own (16.1\%), with friends/peers $(6.5 \%)$, or in supportive/transitional housing $(3.2 \%)$. The primary substance used was identified as cannabis (38.7\%), alcohol $(32.3 \%)$, or another substance $(19.4 \%)$. More than one primary substance was reported by $40 \%$ of participants. The majority (65\%) screened positively for substance-use disorder. Almost half (48\%) endorsed concurrent mental health problems. All participants were attending outpatienttreatment services (eg, individual or group treatment) at least weekly, and 29\% were receiving intensive day treatment. More detailed information about the participants can be found in Goodman et al. ${ }^{2}$

Informed consent was obtained prior to participation in the study. Interviews were conducted individually, and participants received compensation in the form of a CA $\$ 20$ gift certificate for their time. The interviews lasted 45-90 minutes, and were conducted in an interview room in the clinical service area of the hospital by a doctoral student with experience in qualitative data collection. As part of a more comprehensive semistructured interview aimed at understanding various factors affecting youth substanceuse change and treatment engagement, youth were asked the following questions. Tell me about the time in your life when you first thought about changing your alcohol/drug use, and why you began to think about making changes. Did anyone else contribute to your thoughts around changing your substance use? How were they involved? Is there any connection between your substance use and your [friendships, family relationships, etc.]? Was this one of your reasons for changing your substance use and/or seeking treatment?

Interviews were recorded and transcribed verbatim. Identifying information of participants was removed to ensure anonymity and confidentiality. Transcripts were analyzed using thematic analysis, as described by Braun and Clarke. ${ }^{34}$ The primary author engaged in multiple readings of the transcripts to gain familiarity with the data and to look for preliminary patterns and statements concerning 
treatment seeking. Once familiarity with the data had been obtained, first-level coding involved establishing initial codes by highlighting words in the text that captured key concepts and then naming these ideas in the side of the text. A codebook was created to document emerging codes, definitions for each code, and examples from the data. ${ }^{36}$ During the first stage of the coding process, the study investigator and a research assistant independently analyzed seven interview transcripts to identify initial codes. The researchers then reviewed the codes that each had identified. Interrater reliability for the qualitative data was calculated, with results indicating that there was $93 \%$ agreement between the two researchers. Discrepancies in codes were carefully discussed by the raters, and changes were made in the codebook and used for subsequent coding. Second-level coding included examining the data at a broader level by reviewing the initial codes and pasting them into a Microsoft Word document, followed by organizing and grouping them with consideration given to the relationship between codes and themes. ${ }^{34}$ The code piles were each given names to capture the overarching theme or subthemes. These were then reviewed and refined to ensure that they meaningfully represented codes and were discrete. ${ }^{34}$ The transcripts were then analyzed as a whole to ensure no meaning was lost through coding the data. Statements were selected from each theme category to reflect examples of responses.

\section{Results}

The goal of the data analysis was to identify themes that captured youth identification of factors that influenced them to seek or delay treatment for substance-use problems. It became apparent that the factors they identified could be categorized as personal, social, or structural. A thematic analysis of the transcripts identified four themes capturing personal factors that influenced treatment seeking, four themes capturing social factors that influenced treatment seeking, and three themes capturing structural factors that influenced treatment seeking. These are described in the following sections in order from the theme endorsed by the most participants to the least commonly endorsed, with corresponding narratives from participant interviews to depict each theme further.

\section{Personal factors that influenced treatment seeking}

\section{Theme I: Mental and physical health}

Youth reported that the desire to address their mental health problems and enhance their well-being influenced them to seek treatment. One participant stated,
I originally came because of anxiety ... and I guess kind of talking with [my therapist] I realized also that the drinking was rolled into one (youth 1),

and another reported, "The miserable life I was living wasn't what I wanted. It wasn't happiness" (youth 7). Improving physical health was another reason for seeking treatment:

Realizing that I'd gotten to the point that I was just gross and I didn't really take care of myself and ate all the time ... I was sick all the time. I didn't brush my teeth. I didn't shower often (youth 14).

Many youth identified a desire for self-improvement as influencing their decision to get help, specifically: “... wanting to take more control, reaffirm goals, get back to me" (youth 10), and as another youth stated, "I just felt like I was going nowhere and I really wanted to change because I felt like I was meant for a lot more" (youth 29). Participants also noted negative health consequences of their drinking, including accidents, hospitalization, overdosing, delusions, passing out, and getting sick. Many described a period in which they were "trying to cut back" because they were unhappy or "saw it as a bad thing but it was really difficult", which led to needing "some help from professionals to get it done" (youth 25).

Concurrent mental health problems, particularly anxiety and depression, were reported by some participants to have resulted in delays in seeking treatment. Being worried about what others would think of them because they were in treatment, and that they might break down in sessions and embarrass themselves were reported. As one youth described,

It's just being anxious about going ... just not being able to get up and sleeping through alarms and everything. I think that's just me being depressed (youth 13).

Some felt that they needed to address their mental health issues before they could change their substance use:

I guess really getting past any mental issues or having a greater sense of worth to motivate you more and to change things (youth 22).

\section{Theme 2:Academics}

For participants still in high school or postsecondary, academic success influenced some youth to seek treatment because they thought their substance use was getting in the way of their ability to focus on learning:

I don't feel like I'd be able to study if I'm out partying and drinking every night and then my marks wouldn't be the best (youth 16). 
To complete their secondary or postsecondary education faster, youth thought they needed to change their substance use. This was evident by a participant who said, "I just want to get school done, and I'm having a hard time doing that' (youth 12). Doing well in school to get a good job or get into a postsecondary program also prompted some youth's desire to change. This was revealed by a participant who stated, "University is around the corner ... and I won't be ready for it if I could just continue this way" and went on to say,

I don't want to put on the university application 'Yes, [I've been suspended from high school] for smoking marijuana at lunch'. That's embarrassing (youth 8).

\section{Theme 3: Denial or lack of motivation}

Participants held perceptions and feelings about their substance use that kept some from changing or seeking treatment. As one described,

I think it's just really myself and not having enough motivation sometimes, like I know that I don't care that I'm drinking ... I stop caring about changing and I have to like think, remember what I'm supposed to be doing and why ... it's like I'm my own worst enemy I think (youth 1).

Attempting to manage their substance-use problem on their own or to justify it was also reported: "I thought I could deal with it on my own and that I wasn't an addict and I would justify it to myself' (youth 18). For some youth, the benefits of using substances were believed to outweigh the benefits of changing: "Getting more and more addicted, eventually losing sight of wanting to quit" (youth 23), and as another described,

I was like, 'We probably shouldn't continue to use' and all that stuff, but at the same time you really like using, so you don't want to stop using (youth 24).

\section{Theme 4: Finances}

Youth reported that the financial cost of alcohol and drugs was a reason for seeking treatment: "It's just a reason that I cut down smoking, because I didn't have enough money to support it" (youth 6). It was described as a "financial drain" (youth 10), and as one youth noted, "Drugs are expensive, so I don't have the money and I want to save money, so I had to stop" (youth 29). Some participants resorted to stealing drugs or alcohol, or as one reported, "I'd be stealing money from family members and people I didn't even know, just to finance those habits" (youth 14). Guilt was expressed as a result of feeling that their money should be used for other expenses: "I just felt bad. I don't like doing that at all" (youth 13).

\section{Social factors that influenced treatment seeking}

Theme I: Family relationships

Youth described the disapproval of family members regarding their substance use as influencing their treatment seeking. Specific examples included being told they have a problem, they need to stop, and that drugs and alcohol were contributing to an unhealthy lifestyle: "They obviously saw that I was going nowhere ... They weren't very happy about that" (youth 14). Some participants reported being put in control of their own change, as was evident in the following statement:

They sort of said, 'Listen, it's your life. We can't really support [your drug use], and you are going to have to make the changes, because it's your life.' And I said, 'Yeah, that's true' (youth 8).

Other participants reported being pressured into changing, or seeking treatment to make their parents happy:

The changes were not at all for me. They were for them. I wanted to at least give them the sense that I was doing better (youth 5).

Alternatively, participants "wanted to change to better [their] relationships with family members" (youth 32) or sought treatment to reduce their family members' anxiety and fear, as revealed by a participant who said, "I saw the way that it affected them ... I think I scared everybody" (youth 17). Many youth had family members with a substance-use problem, and were motivated to seek help so that they did not experience the same negative consequences of using substances in adulthood. They also recognized that "It's really just a Band-Aid for a bigger problem" (youth 1). Support from family members in the form of encouragement, recognition of progress, and showing interest was identified as helping youth to continue treatment.

\section{Theme 2: Peer relationships}

Youth reported that disapproval by peers was influential in their seeking treatment:

I had a friend who, like, expressed to me that they did not like the way I drank and that they wanted to see me not drink like that. They didn't enjoy spending time with me if I would be like that (youth 5).

Peers were often noted to have identified or initiated treatment services for the youth: "One of my friends that I had made, he suggested that I try to get in ... he tried to help me 
by calling for me" (youth 11). Being rejected by peers was also a reason for changing:

When I was drinking, the friendships were compromised ... It affected that risk of losing friendships ... Not drinking with me, not inviting me to things (youth 21).

Making new friendships that did not revolve around substances and repairing friendships was a reason for seeking treatment:

I felt like ... if I could get it under control, then I could improve my relationships with my friends or even maybe repair relationships that were damaged, and that was what motivated me (youth 5).

Youth also expressed not wanting to experience the negative consequences some of their peers had faced as a result of using substances, such as legal difficulties, incarceration, or residential treatment related to their substance use: "Just seeing how bad it was for them. It was, just, maybe I don't want this" (youth 12). Support from peers was identified as influencing them to continue pursuing help: "My friend was really, really supportive. She said, 'I'm so proud of you for giving this such a huge step"" (youth 14).

\section{Theme 3: Romantic relationships}

For participants who identified themselves as single, many wanted to seek treatment because they felt that their substance use was preventing them from successfully pursuing a romantic relationship:

I feel like it's almost like I'm insecure about my drug use, and so ... I don't want to get involved with anyone, because I don't want to have to deal with anyone telling me that I shouldn't be doing that or feeling pressured to stop (youth 30).

Those who were currently in a relationship wanted to stop out of fear that their partner would break up with them if they did not: "My girlfriend for sure [influenced me to change], 'cause I didn't want her to break up with me" (youth 29). Partners were also noted to make disapproving comments about their drinking:

So it was just like him saying, 'Well, I don't even want to be around you if you're drinking. You're completely crazy to me every time you're drinking' (youth 1).

Action taken by romantic partners included telling participants about services, encouraging them to go, or going with them to appointments. Partners were also noted to offer support:

[My girlfriend] was also supportive, like, 'I know you're going through some shit, but you need to see that this isn't the best way to go about your dealing with stress' (youth 29).

\section{Theme 4: Being around individuals who use} substances

Participants reported that socializing with family members, peers, romantic partners, roommates, and colleagues who regularly used drugs or alcohol in their presence delayed their treatment-seeking process. There was an evident fear in many participants that changing their substance use would have negative consequences on these close and meaningful relationships, including rejection and difficulty relating to others. One male stated,

[My family] wasn't a reason [for changing]. They were more of a hindrance ... we used to do drugs together. We'd all do bongs together: me, my brother, and my dad (youth 7).

Another youth felt that to keep his friends, he needed to keep smoking:

Friends have been one reason why I'm kind of a little apprehensive [about changing], because I see my friends every day to smoke, pretty much (youth 9).

A female participant described doing heroin with her boyfriend, and that

... there would be talk of 'Maybe we should stop doing this, maybe we should change', but nothing would ever really follow through with it (youth 24).

The convenience of and constant exposure to drinking and using drugs with roommates was indicated to be a barrier:

They're really into weed culture. It's easy for me to get high, because they bake. They learn to, like, infuse the weed into different things (youth 11).

Some participants also mentioned that they drank or used drugs to deal with the frustration evoked by their roommates.

\section{Structural factors that influenced treatment seeking}

Theme I: Professionals

Participants identified various types of professionals who influenced them to seek treatment. A social worker or child 
youth worker was most often identified as having guided them to specialized treatment for substance-use problems, even if the youth was not yet convinced that they needed to change. Once in treatment, the mental health professional was often noted to have prompted and maintained their desire to change:

My psychiatrist and therapist both helped me change and they both made me realize that I needed to change, because if I didn't I wasn't going to live a productive and happy life (youth 18).

For many, their family doctor referred them to treatment or made suggestions about where to go; however, as one participant noted, they did "not [have] much involvement beyond that ... just kind of being there" (youth 9). Another youth who was hospitalized for alcohol use stated,

[The emergency doctor] was just very direct in trying to tell me that I was being very dangerous and not behaving good and that I need to put lots of hard time into getting better ... The clarity helped and it made me get my shit together (youth 10).

School counselors, teachers, and principals were also identified as influential in participants' treatment-seeking process. They were noted to have made comments about the youth's substance use, need to stop using, and potential for a bright future, as well as where to go for help.

\section{Theme 2: Dissatisfaction with previous treatment}

Some youth described previous treatment experiences that made them seek treatment somewhere else or delay treatment. Participants reported feeling as though they did not relate to the other treatment-group members or to the issues being discussed in session: "I did not find it helpful to be in a group with kids that can't relate to your situation" (youth 12). Similarly, another youth stated, "I was just ... in a different place than what the group was kind of addressing with certain people" (youth 2). A female participant also described dissatisfaction with the counselors:

I didn't connect with the counselors and I felt that they weren't really necessarily helping me for what I wanted to be helped with (youth 30).

Negative experiences, including “... feeling really judged and kind of manipulated" (youth 23), feeling as though the facilitator "... did not understand how something is painful or kind of brushed aside the issue" (youth 20), and "Finishing a meeting or whatever, it's like I feel worse than I did when I came in" (youth 19) were also reported.

\section{Theme 3:Transport and scheduling}

Logistical arrangements were identified as having delayed pursuing treatment for problematic substance use. As one youth stated, "Transportation is the biggest thing ... it's like a 2-hour commute" (youth 29). Feeling as though they did not have time to come for an appointment because of activities or commitments, such as school and work, and the timing of appointments (eg, too early in the day) were reported as making it hard to seek and continue participating in treatment. When youth were available for a session, it was hard for some, because "The buses don't run very frequently" (youth 9) or they could not afford the bus fare. Another youth reported,

You guys are in kind of an inconvenient spot, so if I was coming by transit I'd probably be less likely to come, but I'm driving, so it's not too bad (youth 26).

\section{Discussion}

The results of this study confirm previous research findings demonstrating negative consequences of substance abuse on personal and social functioning ${ }^{8}$ and the influence of personal, social, and structural factors on the decision to seek treatment for substance use. ${ }^{14,21}$ In addition, these findings extend our knowledge by revealing youth perceptions of influential factors for seeking or delaying treatment that go beyond the internal and external motivators proposed by SDT. Consistently with other studies, ${ }^{11,21,32}$ the primary reason that youth sought treatment was the influence of family members, peers, or romantic partners. This aligns with SDT by reflecting external pressures and the resulting motivation to seek treatment to improve or maintain important relationships. Goodman et $\mathrm{al}^{21}$ found that peer pressure to enter treatment was a significant predictor of motivation to seek treatment and more influential than family pressure for older youth. Youth in the present study more often reported disapproval from family members compared to peers, and support from peers compared to family members, as having influenced them to seek treatment. This is congruent with SDT, and suggests that family members act primarily as external motivators, while peers prompt internal motivation to stay connected to and accepted by peers. As discussed by Wild et al, ${ }^{32}$ internal motivation, or what SDT refers to as self-interest and autonomous drive, may emerge once the youth has committed to the goals of the treatment program. 
Progression toward meeting a goal, observable results, and feedback from the youth's social network appear to predict competence and in turn motivation to stay in treatment, which is consistent with previous findings. ${ }^{26}$ However, it is important to recognize that prominent influences may shift between personal, social, and structural factors throughout the treatment-seeking and treatment-engagement stages.

Factors beyond those encompassed by SDT were also revealed as having influenced youth decisions to seek treatment for substance abuse. These predominantly included structural factors, including satisfaction with previous service utilization, accessibility of services, and availability of clinicians. These findings highlight the importance of considering influences beyond the self and social context, as proposed by SDT, to understand the treatment-seeking process. Going beyond SDT, it is critical to recognize ways to structure health-care services such that youth feel that treatment is accessible and that they will be adequately supported.

The discrepancy between the number of youth with substance-use problems and the number who seek treatment is a prominent concern. ${ }^{11}$ This study provides insight on why this might be the case. Consistent with previous studies of treatment barriers, ${ }^{37}$ youth reported avoiding seeking treatment because of negative views they held toward treatment or their perceived stigma of attending treatment, believing that it would reflect negatively on them. Also consistent with the present findings, ${ }^{38,39}$ lack of flexibility in agency hours and comorbid psychological problems were perceived as making it difficult to access care and keep appointments. In addition, youth in the current study uniquely reported dissatisfaction with the therapist, fellow group members, or program structure as factors leading to their hesitancy to pursue treatment. Given the association between motivation for behavior change and engagement in treatment, ${ }^{32}$ it is critical that service providers find a way to address factors delaying youth treatment seeking, so as to make entering treatment more accessible, less daunting, and more individualized for youth.

Findings from the current study reveal the need for firstcontact professionals (eg, family doctors) who work with youth to be well informed about treatment options when advising youth and referring them for services. Based on the findings of this study, motivational interviewing techniques should be used by professionals, given their utility in enhancing youth awareness of positive reasons for changing and negative reasons for staying the same. ${ }^{40}$ Parents would also benefit from instruction on effective strategies to support their child to seek treatment for their substance use.
It is important that parents attempt to maintain their child's self-determination and autonomy, as opposed to coercing or pressuring them, in the process of thinking about changing and getting help. ${ }^{41}$ While little intervention research exists that elucidates specific SDT-informed approaches for parents of older youth and young adults with substance concerns, such strategies as those included in multidimensional family therapy have been shown to have positive effects on treatment engagement and retention for adolescents with substance-use problems. ${ }^{42,43}$ Similarly, school staff should be encouraged to learn about evidence-based school substance use-prevention curricula, which has been found to have high fidelity for quality of delivery and participant responsiveness. ${ }^{44}$ Cognitive-behavior therapies aimed at sustained change in substance use and behavior have also been found to be effective and easily adaptive for implementation in schools. ${ }^{45}$

There were some variations in the experiences of participants. Indeed, the same factor for some youth was experienced as supporting treatment seeking, while for others it was a hindrance. For example, peers were identified as having expressed concern or offered suggestions about where to go for treatment, yet peers were also noted to have influenced resistance to treatment, because using substances was their primary social activity. These results reveal the importance of service providers gaining an understanding of the complex network of factors influencing a youth's treatment seeking for their substance use, particularly with regard to social factors (eg, peers) and personal factors (eg, comorbid mental health problems). This should involve the youth and professional identifying the circumstances and triggers maintaining their substance use, as well as reasons for seeking treatment to change their substance use.

There are methodological limitations in the present study that should be addressed in future investigations. Interviews were conducted at various points in participants' treatment. Specifically, for some youth, interviews were conducted shortly after intake; for other youth, interviews took place after they had been receiving treatment for a number of months. This may have biased the sample, in that youth who did not seek treatment and those who left treatment early are not in the sample. It may be that youth who had been in treatment for a while were for the most part motivated to change at the time of the interviews, and this could have clouded their memory of why they were previously resistant to changing. Subsequent studies should investigate youth perceptions during the intake process, and should include youth who have not yet sought treatment, as well as those who have dropped out of treatment. It is also possible that 
those who had a more or less challenging treatment-seeking experience did not wish to partake in the research. All participants were obtaining treatment services from one urban treatment facility, and the sample size was relatively small, especially given that it spanned ages typically associated with developmental transitions. Accordingly, limitations to the breadth and generalizability of findings should be considered. Future studies that gather perceptions across various hospital and clinic settings may reveal a more varied experience of seeking treatment, including a broader range of challenges and influential factors.

Despite the limitations of the present study, these findings have added to our understanding of factors influencing youth to seek or delay treatment for problematic substance use. While previous literature has reported on motivations for seeking treatment, this study used SDT to consider the perspective of youth with regard to internal and external influences, as well as structural factors. The breadth and depth of these findings can provide valuable insight for clinicians and service providers to develop strategies to enhance youth desire to change their substance use, make it easier to access treatment early, and engage youth once they have entered treatment.

\section{Acknowledgments}

The authors would like to thank the staff and students who supported the study, and in particular the youth who participated. The authors would also like to acknowledge Dr Lisa Hawke for her support in preparing this manuscript.

\section{Disclosure}

The authors report no conflicts of interest in this work.

\section{References}

1. D'Amico EJ, Ellickson PL, Wagner EF, et al. Developmental considerations for substance use interventions from middle school through college. Alcohol Clin Exp Res. 2005;29(3):474-483.

2. Goodman I, Henderson J, Peterson-Badali M, Goldstein A. Youth perspectives on the transition to adulthood. Emerg Adulthood. 2016; 4(2):92-103.

3. Public Health Agency of Canada. The Human Face of Mental Health and Mental Illness in Canada 2006. Ottawa: PHAC; 2006.

4. Pearson C, Ali J, Janz T. Health at a glance: mental and substance use disorders in Canada. 2013. Available from: http://www5.statcan.gc.ca/ olc-cel/olc. action?ObjId=82-624-X201300111855\&ObjType=47\& lang=en\&limit $=0$. Accessed October 18, 2017.

5. Rush B, Urbanoski K, Bassani D, et al. Prevalence of co-occurring substance use and other mental disorders in the Canadian population. Can J Psychiatry. 2008;53(12):800-809.

6. Hawkins JD, Catalano RF, Miller JY. Risk and protective factors for alcohol and other drug problems in adolescence and early adulthood: implications for substance abuse prevention. Psychol Bull. 1992;112(1): 64-105.
7. National Drug Intelligence Center. The Economic Impact of Illicit Drug Use on American Society. Washington: US Department of Justice; 2014.

8. Ginzler JA, Garrett SB, Baer JS, Peterson PL. Measurement of negative consequences of substance use in street youth: an expanded use of the Rutgers Alcohol Problem Index. Addict Behav. 2007;32(7): $1519-1525$.

9. Dennis ML, Ives ML, White MK, Muck RD. The Strengthening Communities for Youth (SCY) initiative: a cluster analysis of the services received, their correlates and how they are associated with outcomes. J Psychoactive Drugs. 2008;40(1):3-16.

10. Williams RJ, Chang SY. A comprehensive and comparative review of adolescent substance abuse treatment outcome. Clin Psychol Sci Pract. 2000;7(2):138-166.

11. Blanco C, Iza M, Rodriguez-Fernandez JM, Baca-Garcia E, Wang S, Olfson M. Probability and predictors of treatment-seeking for substance use disorders in the U.S. Drug Alcohol Depend. 2015;149: 136-144.

12. Gulliver A, Griffiths KM, Christensen H. Perceived barriers and facilitators to mental health help-seeking in young people: a systematic review. BMC Psychiatry. 2010;10:113.

13. Paglia-Boak A, Adlaf EM, Mann RE. Drug Use among Ontario students - 1977-2011: Detailed OSDUHS Findings. Toronto: Centre for Addiction and Mental Health; 2011.

14. Lewis SF. Examining changes in substance use and conduct problems among treatment-seeking adolescents. Child Adolesc Ment Health. 2013;18(1):33-38.

15. Brown $\mathrm{CH}$, Bennett ME, Li L, Bellack AS. Predictors of initiation and engagement in substance abuse treatment among individuals with co-occurring serious mental illness and substance use disorders. Addict Behav. 2011;36(5):439-447.

16. Rickwood DJ, Deane FP, Wilson CJ. When and how do young people seek professional help for mental health problems? Med J Aust. 2007;187(7 Suppl):S35-S39.

17. Nebhinani N, Sarkar S, Ghai S, Basu D. Reasons for help-seeking and associated fears in subjects with substance dependence. Indian J Psychol Med. 2012;34(2):153-158.

18. Kilpatrick DG, Acierno R, Saunders B, Resnick HS, Best CL, Schnurr PP. Risk factors for adolescent substance abuse and dependence: data from a national sample. J Consult Clin Psychol. 2000;68(1):19-30.

19. Leshner AI. Introduction to the special issue: the National Institute on Drug Abuse's (NIDA's) Drug Abuse Treatment Outcome Study (DATOS). Psychol Addict Behav. 1997;11(4):211-215.

20. Battjes RJ, Gordon MS, O’Grady KE, Kinlock TW, Carswell MA. Factors that predict adolescent motivation for substance abuse treatment. J Subst Abuse Treat. 2003;24(3):221-232.

21. Goodman I, Peterson-Badali M, Henderson J. Understanding motivation for substance use treatment: the role of social pressure during the transition to adulthood. Addict Behav. 2011;36(6):660-668.

22. Loke AY, Mak YW. Family process and peer influences on substance use by adolescents. Int J Environ Res Public Health. 2013;10(9): 3868-3885.

23. Wisdom JP, Cavaleri M, Gogel L, Nacht M. Barriers and facilitators to adolescent drug treatment: youth, family, and staff reports. Addict Res Theory. 2011;19(2):179-188.

24. Rapp RC, Xu J, Carr CA, Lane DT, Wang J, Carlson R. Treatment barriers identified by substance abusers assessed at a centralized intake unit. J Subst Abuse Treat. 2006;30(3):227-235.

25. Misch DA. "Natural recovery" from alcohol abuse among college students. J Am Coll Health. 2007;55(4):215-218.

26. Simoneau H, Bergeron J. Factors affecting motivation during the first six weeks of treatment. Addict Behav. 2003;28(7):1219-1241.

27. De Leon G. Integrative recovery: a stage paradigm. Subst Abuse. 1996; 17(1):51-63.

28. Deci EL, Ryan RM. Intrinsic Motivation and Self-Determination in Human Behavior. New York: Plenum; 1985. 
29. De Leon G, Melnick G, Hawke J. The motivation-readiness factor in drug treatment: implications for research and policy. In: Levy JA, Stephens RC, McBride DC, editors. Advances in Medical Sociology. Greenwich (CT): JAI Press; 2000:103-129.

30. Ryan RM, Deci EL. Intrinsic and extrinsic motivations: class definitions and new directions. Contemp Educ Psychol. 2000;25(1):54-67.

31. Wild TC, Enzle ME. Social contagion of motivational orientations. In: Deci EL, Ryan RM, editors. Handbook of Self-Determination Research. Rochester (NY): University of Rochester Press; 2002:141-159.

32. Wild TC, Cunningham JA, Ryan RM. Social pressure, coercion, and client engagement at treatment entry: a self-determination theory perspective. Addict Behav. 2006;31(10):1858-1872.

33. Henderson J, Cleverley K, Chaim G, Goodman A, Cheung A. Treatment seeking and behaviour change in transition-aged youth with substance use problems: lessons from research. J Can Acad Child Adolesc Psychiatry. 2016;25(Suppl 3):44.

34. Braun V, Clarke V. Using thematic analysis in psychology. Qual Res Psychol. 2006;3(2):77-101.

35. Smith JA. Evaluating the contribution of interpretative phenomenological analysis. Health Psychol Rev. 2011;5(1):9-27.

36. DeCuir-Gunby JT, Marshall PL, McCulloch AW. Developing and using a codebook for the analysis of interview data: an example from a professional development research project. Field Methods. 2011; 23(2):136-155.

37. Cunningham JA, Sobell LC, Sobell MB, Agrawal S, Toneatto T. Barriers to treatment: why alcohol and drug abusers delay or never seek treatment. Addict Behav. 1993;18(3):347-353.
38. Browne T, Priester MA, Clone S, Iachini A, DeHart D, Hock R. Barriers and facilitators to substance use treatment in the rural south: a qualitative study. J Rural Health. 2016;32(1):92-101.

39. Ross LE, Vigod S, Wishart J, et al. Barriers and facilitators to primary care for people with mental health and/or substance use issues: a qualitative study. BMC Fam Pract. 2015;16:135.

40. Naar-King S, Suarez M. Motivational Interviewing with Adolescents and Young Adults. New York: Guildford Press; 2010.

41. Urbanoski KA. Coerced addiction treatment: client perspectives and the implications of their neglect. Harm Reduct J. 2010;7:13.

42. Rowe CL. Family therapy for drug abuse: review and updates 2003-2010. J Marital Fam Ther. 2012;38(1):59-81.

43. Rowe CL. Multidimensional family therapy: addressing co-occurring substance abuse and other problems among adolescents with comprehensive family-based treatment. Child Adolesc Psychiatr Clin N Am. 2010;19(3):563-576.

44. Ennett ST, Haws S, Ringwalt CL, et al. Evidence-based practice in school substance use prevention: fidelity of implementation under real-world conditions. Health Educ Res. 2011;26(2):361-371.

45. Benningfield MM. Meeting youth where they are: substance use disorder treatment in schools. Child Adolesc Psychiatr Clin N Am. 2016; 25(4):661-668.
Patient Preference and Adherence

\section{Publish your work in this journal}

Patient Preference and Adherence is an international, peer-reviewed, open access journal that focuses on the growing importance of patient preference and adherence throughout the therapeutic continuum. Patient satisfaction, acceptability, quality of life, compliance, persistence and their role in developing new therapeutic modalities and compounds to optimize

\section{Dovepress}

clinical outcomes for existing disease states are major areas of interest for the journal. This journal has been accepted for indexing on PubMed Central. The manuscript management system is completely online and includes a very quick and fair peer-review system, which is all easy to use. Visit http://www. dovepress.com/testimonials.php to read real quotes from published authors. 